Conclusion Although oral iron is a cheap and convenient treatment for IDA, over half of the patients in this study experienced gastrointestinal side effects, a large proportion remained anaemic and for many the treatment course was long. Despite iron therapy IDA prevalence in this group remains high and it seems the efficacy of oral iron treatment is poor in this setting. We propose that prescription of iron therapy in IBD patients should be trialled with a defined duration of treatment and a target end point $\mathrm{Hb}$. Those who fail to tolerate or do not respond adequately should consider an alternative form of iron therapy such as IV iron.

Competing interests None declared.

\section{PMO-257 COST-EFFECTIVENESS AND COST COMPARISON OF INTRAVENOUS IRON PREPARATIONS IN PATIENTS WITH IBD-ASSOCIATED IRON DEFICIENCY ANAEMIA BASED ON THE FERGIMAIN TRIAL}

doi:10.1136/gutjnl-2012-302514b.257

${ }^{1} \mathrm{~F}$ S Gutzwiller,* ${ }^{1} \mathrm{P}$ R Blank, ${ }^{2} \mathrm{C}$ Gasche, ${ }^{2} \mathrm{R}$ Evstatiev, ${ }^{1,3} \mathrm{M}$ Schwenkglenks, ${ }^{1} \mathrm{~T}$ D Szucs. IInstitute of Pharmaceutical Medicine (ECPM), Universität Basel, Basel, Switzerland; '2Division of Gastroenterology \& Hepatology, Department of Medicine 3, Medical University of Vienna, Vienna, Austria; ${ }^{3}$ Institute of Social and Preventive Medicine, University of Zurich, Zurich, Switzerland

Introduction Anaemia is a costly complication of IBD and a common trigger of hospitalisation. The FERGImain trial, a multi-centre, randomised, placebo-controlled study, demonstrated that ondemand ferric carboxymaltose (FCM) reduced recurrence of anaemia in non-anaemic IBD patients, which received either FCM or placebo when ferritin-levels fell $<100 \mu \mathrm{g} / \mathrm{l}$. Time to anaemia was assessed within 8 months. We present economic results for this trial and compare costs with those expected for the use of low molecular weight iron dextran (ID) from the perspective of the UK National Health Service.

Methods Patients were analysed when at least one haemoglobin value at baseline and a subsequent value was available (204 randomised and treated (105 FCM, 99 placebo)). Trial-based information on medical resource use was combined with UK prices for drugs, wages and materials. Starting from the single administered dose of FCM (500 mg within $15 \mathrm{~min}$ ) a comparative cost-analysis was performed against ID (500 mg within $4 \mathrm{~h}$ ). Incremental costs per avoided anaemia case were calculated.

Results Time to recurrence of anaemia in patients treated for low ferritin was significantly longer in the FCM group (HR 0.62 [95\% CI 0.38 to 1.00$]$, Kaplan-Meier analysis; $\mathrm{p}=0.049$, log-rank test). By month 8, anaemia had recurred in $26.7 \%$ (FCM) and $39.4 \%$ (placebo) of patients. Total costs (including hospitalisation) added up to $£ 499$ ( $\pm 1453, \mathrm{SD})$ in the FCM arm and $£ 319$ for placebo ( $\pm 1390 \mathrm{SD})$. The average cost per avoided anaemia case over the study period was $£ 1414$. Drug and administration costs for FCM totalled to $£ 231$ ( $£ 189$ and $£ 41$, respectively), for a mean cumulative dose of $990 \mathrm{mg}$ iron administered per patient over the study period. Compared to FCM, drug and administration costs for an equal dose of ID would be $£ 517$ ( $£ 79$; £438), respectively.

Conclusion Prevention of anaemia recurrence with FCM is effective and associated with additional costs. However, as treatment costs for single-anaemia episodes are not available, our data on costs per avoided anaemia case is difficult to interpret. In comparison, yearly costs in 2001 for a single anaemic IBD patient have been estimated to $£ 7442$ ( $\$ 10687$ ). The hypothetical use of ID may save drug costs on the expense of administration costs.
Competing interests F Gutzwiller: Grant/Research Support from: Vifor Pharma Ltd, Switzerland, Conflict with: received a travel grant from Vifor Pharma Ltd, Switzerland, P Blank: Grant/Research Support from: Vifor Pharma Ltd, Switzerland, C Gasche: Conflict with: grant and consultancy honoraria from Vifor International, Pharmacosmos A/S, Fresenius Medical Care, Renapharma Sweden, R Evstatiev: None declared, M Schwenkglenks: Grant/Research Support from: Vifor Pharma Ltd, Switzerland, T D Szucs: Grant/Research Support from: Vifor Pharma Ltd, Switzerland.

\section{PM0-258 UNDER THE MICROSCOPE: A REVIEW OF THE MANAGEMENT OF MICROSCOPIC COLITIS ACROSS NORTH EAST ENGLAND}

doi:10.1136/gutjnl-2012-302514b.258

F P Porras Perez.* Department of Gastroenterology, University Hospital North Tees, Stockton-on-Tees, UK

Introduction Microscopic colitis (MC) encompasses lymphocytic (LC) and collagenous colitis (CC) and typically presents with chronic watery diarrhoea. This retrospective review of biopsy proven cases from 5 DGH reports the clinical characteristics, possible drug precipitants, treatment and outcome in 49 cases.

Methods Retrospective review of patients with histopathological confirmation of MC over a 6-month period between July 2009 and December 2009 across two FTs (5 DGHs). Demographics, clinical presentation including suspected precipitant drugs, investigations, treatment, evolution, and screening for AI diseases were collected. For patients discharged from hospital follow-up, their GPs were contacted to evaluate ongoing symptoms.

Results 49 patients ( 38 females and 11 males) fulfilled the diagnostic criteria for MC (15 LC and 34 CC). Mean age 66 years (34-90). Mean symptom duration prior to diagnosis was 4.6 months (1-24 months). Main symptoms at the time of diagnosis; diarrhoea (100\%), abdominal pain (24.4\%), weight lost (6.1\%) and faecal incontinence $(8.1 \%)$. Diagnosis made by flexible sigmoidoscopy $(38.7 \%)$ or colonoscopy (61.3\%). All patients offered clinic FU (4 DNA). Endoscopic findings reported as grossly normal in all cases. 41 patients were discharged back to GP care and 8 were under active FU in clinic. Of the 41 discharged patients, 10 had at least 1 further flare up of symptoms within 1 year of being discharged. 16 patients were treated pharmacologically: 3 with 5-ASA and 13 with oral Budesonide; 13 had an initial remission and in 6 remained symptomatic at the time of this review. 31 patients $(63.26 \%)$ did not receive therapy and eight of those reported recurrent flare ups. 36 patients $(73.46 \%)$ had received drugs reported to be associated with LC at some point and in 22 (61.11\%) these had not been discontinued; of these, 7 (22.58\%) reported at least one flare up. By registering all medications at diagnosis we found 22 patients were on PPIs, 12 patients on NSAIDs and 8 patients on SSRIs. Coeliac serology was checked in 27 patients and was positive in one patient. 12 patients $(24.48 \%)$ gave a history of $\mathrm{AI}$ diseases.

Conclusion In the absence of specific guidelines, management of MC was highly variable. Only $55.1 \%$ of cases were screened for coeliac disease and possible drug precipitants were not routinely detected and in $61.11 \%$ of cases these drugs were not discontinued. Gross endoscopic appearances were normal in all cases, reinforcing the need to take colonic biopsies in all cases of unexplained diarrhoea. While the majority of patients were discharged from follow-up, a significant number had re-attended in primary care with recurrent symptoms suggesting the disease burden may be underestimated. Specific national guidelines for the investigation, treatment and follow-up of MC would be valuable.

Competing interests None declared. 